\title{
Alpha/Beta T-Lymphocyte
}

National Cancer Institute

\section{Source}

National Cancer Institute. Alpha/Beta T-Lymphocyte. NCI Thesaurus. Code C156387.

A mature T-cell that expresses a T-cell receptor complex comprised of the highly variable alpha and beta chains complexed with the invariant CD3 antigen complex and plays a primary role in adaptive immunity. 